\title{
Educación para el cambio climático: ¿Por qué formar para afrontar la incertidumbre, vulnerabilidad y complejidad ambiental?
}

\author{
Climate Change Education: Why to Train to Cope With Environmental Uncertainty, \\ Vulnerability and Complexity?
}

\section{Educação sobre mudanças climáticas: Por que treinar para lidar com a incerteza ambiental, vulnerabilidade e complexidade?}

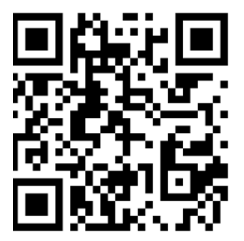

Jesús Núñez-Rodríguez

Universidad de Santander Facultad de Ciencias Económicas

Administrativas y Contables Grupo de Investigación Ciempiés

Cúcuta, Colombia

jo.nunez@mail.udes.edu.co

https://orcid.org/0000-0002-4120-0215

Recibido • Received • Recebido: 03 / 07 / 2019

Corregido • Revised • Revisado: 25 / 02 / 2021

Aceptado • Accepted • Aprovado: 06 / 04 / 2021

\begin{abstract}
Resumen: El objetivo de este ensayo es analizar los conceptos de incertidumbre, vulnerabilidad y complejidad ambiental en los escenarios del cambio climático presente y futuro, y el imprescindible rol de la escuela en formar una ciudadanía con capacidades para su mitigación y adaptación. Pensando en los riesgos para la especie humana y en la apuesta en la educación, este artículo está dirigido al personal docente, y a sus estudiantes, con el propósito de incorporar nuevos constructos en las competencias cognitivas, axiológicas y procedimentales como herramientas útiles para sobrevivir en ambientes vulnerables, complejos e inciertos. La metodología en la construcción del ensayo se basó en la revisión de los conceptos analizados en los estudios desarrollados por los organismos internacionales relacionados con la ciencia climática, en investigaciones que reportan evidencias en revistas indexadas y su posterior valoración de aplicación en los procesos educativos. Como una síntesis de la discusión realizada se concluye la preocupante exposición de la sociedad humana contemporánea a cambios drásticos en el clima que aumentan la vulnerabilidad de la población en situación de pobreza, al afectar la producción de alimentos, disponibilidad de agua potable, incidencia de plagas y enfermedades, y la pérdida de vidas humanas en eventos climáticos extremos. En este escenario, la educación se erige como una de las instituciones llamadas a formar a las nuevas generaciones para sobrevivir en un planeta más caliente y con un mapa demográfico desconocido por las graduales migraciones climáticas en búsqueda de recursos de agua, alimentos, suelos y climas vivibles.
\end{abstract}

Palabras claves: Cambio climático; incertidumbre ambiental; vulnerabilidad ambiental; complejidad ambiental; educación. 
http://doi.org/10.15359/ree.25-2.28

http://www.una.ac.cr/educare

educare@una.ac.cr

\begin{abstract}
The objective of this essay is to analyze the concepts of environmental uncertainty, vulnerability, and complexity in the scenarios of present and future climate change, and the essential role of the school in forming citizens with capacities for mitigation and adaptation. As this article takes into consideration the bet on education and the risks for the human species, it is addressed to teachers and their students to incorporate new constructs in cognitive, axiological, and procedural competencies as useful tools to survive in vulnerable, complex, and uncertain environments. The methodology to write the essay was based on the review of the concepts analyzed in studies conducted by international organizations related to climate science, and by researchers who report evidence in indexed journals and their subsequent assessment of applications in educational processes. As a synthesis of the discussion carried out, we conclude that the contemporary human society is worryingly exposed to drastic climate changes that increase the vulnerability of the poor population by affecting food production, availability of drinking water, the incidence of pests and diseases, and loss of human lives in extreme climate events. In this scenario, education emerges as one of the institutions called to train new generations to survive on a warmer planet and with an unknown demographic map due to gradual climate migrations in search for water, food, soil, and livable climates.
\end{abstract}

Keywords: Climate change; environmental uncertainty; environmental vulnerability; environmental complexity; education.

Resumo: $O$ objectivo deste ensaio é analisar os conceitos de incerteza, vulnerabilidade e complexidade ambiental nos cenários de alterações climáticas presentes e futuras e o papel essencial da escola na formação de cidadãos com capacidades para a sua mitigação e adaptação. Pensando nos riscos para a espécie humana e na aposta na educação, este artigo é dirigido aos professores e seus alunos, com o objectivo de incorporar novas construções em competências cognitivas, axiológicas e processuais como ferramentas úteis para sobreviver em ambientes vulneráveis, complexos e incertos. A metodologia na construção do ensaio baseou-se na revisão dos conceitos analisados nos estudos desenvolvidos por organizações internacionais relacionadas com as ciências climáticas, em investigadores que relatam provas em revistas indexadas e na sua subsequente avaliação da aplicação em processos educativos. Como síntese da discussão realizada, concluímos a preocupante exposição da sociedade humana contemporânea a mudanças drásticas no clima que aumentam a vulnerabilidade da população pobre, afectando a produção alimentar, a disponibilidade de água potável, a incidência de pragas e doenças, e a perda de vidas humanas em eventos climáticos extremos. Neste cenário, a educação ergue-se como uma das instituições chamadas a formar novas gerações para sobreviver num planeta mais quente e com um mapa demográfico desconhecido devido às migrações climáticas graduais em busca de recursos hídricos, alimentos, solo e climas habitáveis.

Palavras-chave: Alterações climáticas;incerteza ambiental;vulnerabilidadeambiental;complexidade ambiental; educação.

\title{
Introducción
}

Las prospectivas negativas del cambio climático ameritan repensar la educación. Se trata de escenarios futuros complejos e inciertos que requerirán de una profunda resiliencia biológica, emocional y cognitiva de la población para la mitigación y adaptación a condiciones climáticas extremas. Es una responsabilidad de la educación de hoy comenzar a apropiarse de

2

Jesús Núñez-Rodríguez

Los artículos de la Revista Electrónica Educare del Centro de Investigación y Docencia en Educación de la Universidad Nacional, Costa Rica, se comparten bajo términos de la Licencia Creative Commons: Reconocimiento, № Comercial, Sin Obra Derivada 3.0 Costa Rica. Las autorizaciones adicionales a las aquí delimitadas se pueden obtener en el correo: educare@una.cr 
nuevos constructos teóricos para ir formando al estudiantado para vivir en contextos cargados de alta incertidumbre ambiental.

La ciencia del cambio climático está generando continuamente información sobre las tendencias del clima, la acumulación de gases de efecto invernadero en la atmósfera y los impactos de este fenómeno sobre la vida de las poblaciones. La Organización de las Naciones Unidas (ONU) especialmente a través del Panel Intergubernamental de Cambio Climático (IPCC) ha advertido que en el año 2100 se podrían superar los límites de $+1,5^{\circ} \mathrm{C}$ y $400 \mathrm{ppm}$ de $\mathrm{CO}_{2}$, por encima de los niveles preindustriales previstos para finales del siglo (Intergovernmental Panel on Climate Change [IPCC], 2019), hito fundamental que denota la gravedad del futuro climático del planeta.

Asimismo, en todo el globo, diversas instituciones y equipos académicos realizan investigaciones sobre la modelización del comportamiento climático y sobre los efectos en la vida de ecosistemas naturales y humanos producto de los cambios en los parámetros de temperatura, precipitación y humedad; todas ellas concluyen en la severidad del aumento de la temperatura y olas de calor (Lopez et al., 2018), sequias e inundaciones (Martín-Ortega, 2011) sobre sujetos concretos y comunidades locales, lo cual generará problemas de enfermedades, escasez de alimentos, migraciones climáticas (Rodríguez de Luque et al., 2016), disminución en la productividad agropecuaria (D’Agostino y Schhlenker, 2016), daños a infraestructuras y servicios (Gourdji et al., 2016), entre otros.

Ahora bien, se observa una brecha importante entre la información derivada del trabajo realizado por las instituciones medioambientales y de investigación académica con respecto al conocimiento, percepciones y representaciones sociales que la población afectada tiene sobre el cambio climático que no permite su pronta apropiación para el acometimiento de acciones de mitigación y adaptación (Kgosikoma et al., 2018). Es aquí donde la escuela debe aprovechar su carácter históricamente mediador para poner en contacto el conocimiento de la ciencia climática con las realidades presentes y futuras de escenarios climáticos signados por los cambios medioambientales.

Con esa pretensión, este ensayo se enfoca en poner a disposición del personal docente algunos constructos teóricos derivados de diferentes disciplinas del conocimiento, en conexión con lo ambiental y lo educativo, para señalar aspectos no valorados en la escuela contemporánea y los cuales consideramos pueden ser de utilidad para la formación de la población en tiempos de cambio climático.

\section{La incertidumbre climática}

Los tiempos de los calendarios escolares planificados de acuerdo con los ciclos productivos y climáticos estandarizados han entrado en crisis por los cambios intempestivos originados por los fenómenos medioambientales y sus efectos colaterales sobre la asistencia, permanencia y desempeño del estudiantado y de sus docentes a las jornadas de clase. 
http://doi.org/10.15359/ree.25-2.28

http://www.una.ac.cr/educare

educare@una.ac.cr

A finales del siglo XX, apenas casi tres décadas, se recalcó el agotamiento del modelo de la modernidad, el fin de un tiempo enmarcado dentro de un proyecto de desarrollo económico enfocado en la ciencia y la tecnología y en la energía fósil, con la promesa de progreso indefinido. Previamente, los resultados del Informe Brundtland dejaron al descubierto, por los años 80, no solo la ampliación y profundización de las brechas sociales entre sectores pobres y ricos (Wyczykier, 2017), sino que nos restregó el rostro nefasto de la destrucción ambiental. En menos de 200 años y de solo 8 generaciones humanas acabamos con las condiciones apropiadas para la vida en la biosfera, revirtiendo la tendencia natural de enfriamiento de la tierra (Marsicek et al., 2018).

La incertidumbre se hace presente cuando en los marcos cognitivos y emocionales no existe la certeza de la ocurrencia de un fenómeno natural o social históricamente recurrente, lo cual impide asumir comportamientos y decisiones seguras en los niveles de confort cotidianos. Al cambiar las condiciones de base del clima conocido se generan percepciones y representaciones sociales del cambio climático por la "imposibilidad de predecir qué eventos puntuales a corto plazo y de carácter local están influenciados, en mayor o menor medida, por el cambio en el clima" (Pancorbo Jiménez, 2012, p. 342); ello se torna en presiones sobre los grupos sociales por las transformaciones significativas que comienzan a manifestarse en los procesos relacionados con la estructura, organización y funcionamiento del entorno medioambiental inmediato al sujeto.

La comprensión conceptual de la incertidumbre climática no es sencilla para la mayoría de la población, pues está asociada a la incapacidad de predecir el comportamiento climático futuro, pues evidencia confusiones por la ocurrencia paralela de fenómenos magnificados de Iluvia-sequia, calor-frio, lluvia-hielo, no tradicionales en el imaginario colectivo. En este ámbito, el IPCC (2005) establece una tipología de incertidumbre climática con base en tres componentes que posibilitan su estudio: a) Impredictabilidad por la dificultad inicial de predecir el comportamiento futuro del clima (nivel del saber cotidiano). b) Incertidumbre estructural, derivada de las limitaciones en los modelos o marcos conceptuales utilizados (nivel epistémico). c) Incertidumbre de valores por la no representatividad de los datos en cuanto a lo espacial o temporal (nivel operativo).

A nivel de la escuela y de la población en general, pareciera que el comportamiento personal y profesional aún se encuentra rígido y encapsulado en las certezas y en el convencimiento de que nada ha cambiado y que podemos seguir planificando, desarrollando y explotando recursos y servicios ambientales como si estos fuesen infinitos, inalterables e inagotables, con una concepción antropocéntrica y eminentemente crematística.

La nueva realidad, cada vez más incierta, obliga al personal educativo (de cualquier nivel del sistema educativo) a la interpretación de la incertidumbre compleja (Soteriades et al., 2017) en entornos medioambientales cambiantes, particularmente influenciados por el cambio climático en sus dos componentes principales: precipitación y temperatura. Los informes del IPCC y de la

4

Los artículos de la Revista Electrónica Educare del Centro de Investigación y Docencia en Educación de la Universidad Nacional, Costa Rica, se comparten bajo términos de la Licencia Creative Commons: Reconocimiento, № Comercial, Sin Obra Derivada 3.0 Costa Rica. Las autorizaciones adicionales a las aquí delimitadas se pueden obtener en el correo: educare@una.cr 
Convención Marco para el Cambio Climático de la ONU evidencian la relación entre el aumento sostenido de la temperatura desde la época preindustrial (antes de 1750) y la acumulación de dióxido de carbono $\left(\mathrm{CO}_{2}\right)$ en la atmósfera, en las cercanías de superar el incremento de los $2{ }^{\circ} \mathrm{C}$ (establecido como límite superior en la COP21 realizada en Francia en 2015). Por primera vez, en la historia humana se alcanzaron las 400 ppm de este gas en la atmosfera, lo cual viabiliza, a futuro, escenarios climáticos ( + de $4{ }^{\circ} \mathrm{C}$ ) de gran incertidumbre y riesgos difíciles de anticipar y planificar (Kammen, 2013).

La incertidumbre climática desconfigura los cronogramas en la planificación, la gradualidad y la alternancia en la ocurrencia de los períodos secos y húmedos que permitían prever con antelación las inversiones y el desarrollo de proyectos; se observan cambios abruptos entre sequias asfixiantes y atroces inundaciones, cuya combinación nefasta deja a su paso enormes pérdidas económicas, ecológicas y sociales por los desastres naturales que provocan los deslizamientos, inundaciones, terremotos (Febriani y Lokantara, 2017), huracanes, tornados, avalanchas, tormentas eléctricas, olas de calor e incendios forestales.

Educar en condiciones de alta incertidumbre climática, generada por múltiples factores exógenos interconectados de difícil reconocimiento y pronóstico (Iglesias, et al., 2011), amerita el monitoreo constante de las variables climáticas locales y la consideración de las prospectivas medioambientales en diferentes escenarios, pensando en la resiliencia futura de las obras de infraestructura y en la población usuaria, más que en la efectividad cortoplacista, mediante la utilización de herramientas de análisis de decisiones y métodos de optimización dinámica para enfrentar la incertidumbre (Williams y Johnson, 2013) en sistemas de alta complejidad ambiental y humana.

La escuela, por consiguiente, debe flexibilizar su forma de organizar y operar sus currículos, de manera que garanticen la prosecución del estudiantado aislado geográficamente o afectado por los eventos climáticos extremos; adaptar los calendarios académicos a nuevos ciclos productivos, a formas alternas de enseñanza y al manejo de la resiliencia biológica, emocional y cognitiva ante situaciones imprevistas, por los desplazamientos de los grupos humanos al interior de sus territorios o fuera de sus países (migraciones climáticas).

\section{La complejidad ambiental}

El mundo es cada día un lugar más complejo y la escuela específicamente es un sistema que reproduce en sus aulas esa realidad por los encuentros y desencuentros de múltiples actores, recursos y experiencias, en íntima imbricación, que denotan los cambios que vienen ocurriendo en la sociedad. La apropiación académica pertinente de las concepciones enmarcadas en los sistemas de comportamiento complejo y de la complejidad social (Supadli et al., 2018), características de los escenarios de cambio climático, impulsa a la interacción de sujetos complejos en la formación prospectiva del estudiantado para realidades climáticas 
http://doi.org/10.15359/ree.25-2.28

http://www.una.ac.cr/educare

educare@una.ac.cr

inciertas. Por lo tanto, requiere, como escenario de mediación pedagógica, participación de la escuela para la consolidación de competencias personales y profesionales para adaptarse al clima del futuro.

Hoy el mundo es un entramado de relaciones complejas, cambiantes e inciertas (Leff, 2007). Esta trilogía epistémica subsume al ser humano y a su entorno en entidades interdependientes, en su interior y exterior, con fronteras difusas y con gran dinamismo al construir, deconstruir y reconstruir continuamente sus procesos fundacionales y estéticos. Profesionales de la docencia, dentro de un ambiente complejo, deben abandonar sus aferraciones disciplinarias de la vieja escuela que no permiten ver, y menos incursionar, en otras áreas del saber y de la experiencia. Es bien notorio que la juventud, hoy, es cada vez más transdisciplinaria, pues las presiones de la sociedad, la alta disponibilidad de información en las redes sociales y la naturaleza de los empleos empujan a estar continuamente buscando nuevos campos de acción dentro de múltiples ámbitos del saber, generalmente ajenos a su formación profesional de base, dentro de nuevas racionalidades del pensamiento transdisciplinar y complejo (Osorio García, 2012) y de la reapropiación social de la naturaleza (Leff, 2004).

En tiempos de cambio climático se debe comprender que las respuestas del planeta ante el calentamiento global no son lineales (causa-efecto) (Kammen, 2013), sino que se encuentran matizadas por una cascada de incertidumbre que plantea un gran desafío (Soteriades et al., 2017), para mitigar y adaptar los impactos en los sistemas naturales y sociales complejos, retos que obligan a diseñar programas y proyectos educativos enmarcados en enfoques de la complejidad social (Supadli et al., 2018), la planificación de la conservación (Williams y Johnson, 2013), de la agricultura climáticamente inteligente (Saj et al., 2017), buenas prácticas agrícolas (Peixoto et al., 2017), la Iniciativa 4 por 1000 de CGIAR System Organization para secuestrar carbono en los suelos (Chabbi et al., 2017), pagos por servicios ambientales (PSA) (Engel y Muller, 2016), entre muchas iniciativas.

La complejidad ambiental signada por la incertidumbre climática y la alta vulnerabilidad de los territorios y poblaciones (constructo siguiente) ponen al personal educativo en el camino desafiante de repensar sus saberes y sus valores para leer e interpretar una realidad compleja (y esquiva), y formar prospectivamente en la consolidación de capacidades creativas e innovadoras en sus estudiantes, para actuar eficientemente en ambientes con climas más calientes, déficit de agua, olas de calor, desastres naturales, nuevas plagas y enfermedades, negocios emergentes..., otros ecosistemas, es decir, un paisaje demográfica y geográficamente diferente al conocido hasta hoy.

\section{La vulnerabilidad ambiental}

Miles de niños y niñas en el mundo ven cómo sus escuelas son destruidas por desastres naturales que afectan a sus comunidades, y las pérdidas infantiles por muerte o desplazamientos por los efectos de las inundaciones o sequías extremas es muy alta. La escuela en tiempos del

Jesús Núñez-Rodríguez

Los artículos de la Revista Electrónica Educare del Centro de Investigación y Docencia en Educación de la Universidad Nacional, Costa Rica, se comparten bajo términos de la Licencia Creative Commons: Reconocimiento, No Comercial, Sin Obra Derivada 3.0 Costa Rica. Las autorizaciones adicionales a las aquí delimitadas se pueden obtener en el correo: educare@una.cr 
cambio climático debe ser una institución inteligente que reflexione y aprenda continuamente a disminuir la alta vulnerabilidad humana y ambiental para desarrollar estrategias de mitigación y adaptación que reduzcan la vulnerabilidad al calor y lluvias extremas (Wilhelmi y Hayden, 2010), y garantizar su funcionamiento y la vida de sus estudiantes. Un clima más caliente traerá condiciones que afectarán la salud de la niñez y la juventud, la disponibilidad de los recursos en sus territorios, su sostenibilidad y las condiciones apropiadas de confort en las aulas de clase para desarrollar los procesos de mediación pedagógica. Este hecho se agudizará en los grupos sociales vulnerables como indígenas, campesinado y residentes en áreas urbano marginales.

Al cambiar las condiciones climatológicas globales descubrirnos la alta vulnerabilidad humana y ecológica, como producto del forzamiento antropogénico (Nangombe et al., 2018), ante los eventos climáticos extremos que perturban el comportamiento y funcionamiento de los núcleos sociales de las regiones biogeográficas frágiles. Vidas humanas, sistemas sociales, sistemas productivos, infraestructuras y servicios de apoyo son destruidos o afectados en cortos períodos de tiempo.

La vulnerabilidad se inscribe dentro de un concepto multidimensional, pues abarca los componentes humanos, naturales y culturales que se evidencian en la capacidad disminuida de la población para anticipar, sobrevivir, resistir y recuperarse de los efectos o amenazas de riesgos de eventos extraños (Bataglia, 2008). El grado de exposición al riesgo y las condiciones de fragilidad socionatural de las personas determinan los impactos negativos de los eventos extremos. En correspondencia, la Comisión Económica para América Latina y el Caribe (CEPAL) establece que existe"vulnerabilidad cuando ocurre un evento adverso y no se tiene la capacidad de respuesta ni la habilidad para adaptarse al nuevo escenario generado" (Durán Gil, 2017, p. 14)

En los escenarios del cambio climático se están presentando efectos adversos sobre poblaciones con alta vulnerabilidad social, ubicadas en los cordones de miseria urbana y rural, con deficientes servicios e infraestructuras básicas, grupos etarios sensibles y bajos niveles de educación. En este último aspecto, Bataglia (2008) emplea el término vulnerabilidades educativas al identificar a grupos significativos de estudiantes de escuelas rurales y urbanas con grandes desigualdades económicas y sociales, asentados en territorios de alta vulnerabilidad, que son perturbados por la incidencia de los fenómenos climáticos intensos.

En estos sectores coexisten dos problemas que deben ser focos de atención del educador o educadora: la salud y la seguridad agroalimentaria.

En la primera, la salud de la población está siendo mermada por la alta incidencia de insectos vectores de virus de enfermedades (malaria, dengue, chikunguya, zika...); afecciones gastrointestinales (diarreas), respiratorias y dérmicas producidas por efecto de las lluvias y las sequias -estos problemas colapsan los sistemas sanitarios y de higiene familiar, por los efectos nocivos en la desnutrición, la salud mental, y en enfermedades que se transmiten por medio de 
http://doi.org/10.15359/ree.25-2.28

http://www.una.ac.cr/educare

educare@una.ac.cr

los alimentos y del agua (UN CC: Learn y Organización Mundial de la Salud, 2013)-, y a la alta vulnerabilidad social a los eventos de estrés fisiológico y mental por el calor extremo (Wilhelmi y Hayden, 2010).

La segunda, se origina por los impactos del cambio climático sobre los sistemas de producción agrícola y de seguridad alimentaria (Rodríguez de Luque et al., 2016), al mermar o destruir plantaciones agrícolas, explotaciones de animales, vías de comunicación, sistemas de riego, disponibilidad, acopio y distribución de los alimentos. Los impactos encarecen los productos, disminuyen la cantidad y calidad, y limitan la accesibilidad de la población a los productos agroalimentarios esenciales (Núñez et al., 2018).

Para mitigar la vulnerabilidad ambiental es pertinente comenzar a desarrollar tecnologías para evaluar los impactos del cambio climático en los campos de la salud, el agua, los bosques, la agricultura, los ecosistemas y los desastres naturales (Chae y Lee, 2018) y; minimizar las emisiones de gases de efecto invernadero ocasionados por el modelo actual de producción actual de alimentos, para lo cual es necesario introducir cambios en los sistemas de producción agrícola y en los hábitos de consumo de alimentos (Graffeo, 2017), y en el modelo energético fósil (carbón y petróleo) por formas sostenibles y de bajo impacto ambiental.

La escuela debe educar para coadyuvar en la vulnerabilidad social ante los eventos extremos. Para ello debe formar competencias prácticas de adaptación, en sus estudiantes, en aspectos básicos de sobrevivencia, tales como primeros auxilios, logística humanitaria, rescate de personas, tratamiento de aguas, organización comunitaria, sistemas de alertas tempranas, georreferenciación, entre muchas. Asimismo, es importante la formación de competencias, en el estudiantado, para la mitigación de los efectos del cambio climático en la regeneración ambiental de ecosistemas destruidos: entre ellas la implementación de buenas prácticas de uso del agua, la recuperación de las nacientes hídricas, el manejo de desechos líquidos y sólidos, la siembra de árboles de sombra alrededor de las escuelas, el uso de energías alternativas, la protección contra el ataque de plagas y enfermedades en las familias y el cuidado a las exposiciones de los rayos solares

De igual manera, el manejo del estrés y la salud mental de docentes y estudiantes requerirán de acciones de mitigación relacionadas con ambientes físicos más abiertos, turnos escolares más flexibles, uso de métodos de alternancia de clases, alimentación balanceada y actividad física adaptada al comportamiento climático.

\section{Consideraciones finales}

La gran complejidad ambiental generada por los efectos del calentamiento global impacta significativamente a la educación, al convertir, gradualmente, los territorios y sus sistemas escolares en espacios entrópicos cambiantes donde interaccionan factores multidimensionales 
emergentes $y$, seguramente, desconocidos en el imaginario del ser humano, y que implican, para el personal docente, la apertura cognitiva y emocional para formar-se en la mitigación y adaptación a los efectos negativos propios de un planeta más caliente. La escuela como institución mediadora del conocimiento debe convertirse en una escuela que aprende y enseña un pensamiento complejo y divergente, para formar competencias en el estudiantado, que le permitan convivir y desarrollarse sosteniblemente en territorios signados por las continuas transformaciones medioambientales y humanas.

La desaparición de las certezas climáticas en una educación históricamente afincada en ciclos regulares del comportamiento atmosférico, que permitían su habitual predicción y planificación pedagógica, generará enormes conflictos en la toma de decisiones en los niveles organizacionales y operativos del sistema escolar. La escuela tradicional de calendario único tendrá que adoptar -y adaptar-, en el escenario del cambio climático, metodologías gerenciales y didácticas contingentes para adecuar su organización y funcionamiento a la coexistencia de nuevos equilibrios climáticos en convivencia con la incertidumbre en la ocurrencia de fenómenos medioambientales extremos. Una educación para la incertidumbre climática debe formar competencias en sus estudiantes para "leer" e interpretar dinámicamente su entorno climático para actuar proactivamente ante los cambios que afectarán su desarrollo cognitivo, emocional y productivo.

La fragilidad de los territorios y grupos humanos, en gran parte del planeta, es un factor que potencia negativamente los impactos de la acción del cambio climático. En el ámbito escolar, la vulnerabilidad educativa representa una amenaza real de incrementar la deserción estudiantil por la pobreza y la migración de poblaciones por motivos climáticos. Una educación para mitigar la vulnerabilidad ambiental y adaptar la escuela a ello, especialmente en territorios sensibles y grupos humanos económicamente deprimidos, debe impulsar la implementación de políticas públicas de reforzamiento de las capacidades locales de seguridad agroalimentaria y cuidados de la salud; la formación de competencias en el estudiantado para el aprovechamiento de las nuevas condiciones medioambientales $y$, el desarrollo de habilidades para la resiliencia biológica, emocional y cognitiva en ambientes complejos, inciertos y vulnerables.

\section{Declaración de Material complementario}

Este artículo tiene disponible, como material complementario:

-La versión preprint del artículo en https://doi.org/10.5281/zenodo.3922555 
http://doi.org/10.15359/ree.25-2.28

http://www.una.ac.cr/educare

educare@una.ac.cr

\section{Referencias}

Bataglia, M. A. (2008). Vulnerabilidad educativa, política e institucional en comunidades afectadas por las inundaciones de llanuras: Aspectos fundamentales para la gestión y la gobernabilidad. Revista Geográfica Digital Igunne, 5(10), 2-19. https://doi.org/10.30972/ geo.5102828

Chabbi, A., Lehmann, J., Ciais, P., Loescher, H. W., Cotrufo, M. F., Don, A., SanClements, L., Schipper, J., Six, P., Smith y Rumpel, C. (2017). Aligning agriculture and climate policy. Nature climate change, 7(5), 307-309. https://doi.org/10.1038/nclimate3286

Chae, S. H. y Lee, M. J. L. (2018). Building of platform for development of integrated model to assess climate change impacts and vulnerability. IOP Conference Series: Earth and Environmental Science, 151(1), 1-7. https://doi.org/10.1088/1755-1315/151/1/012004

D’Agostino, A. L. y Schlenker, W. (2016). Recent weather fluctuations and agricultural yields: implications for climate change. Agricultural Economics, 47(S1), 159-171. https://doi. org/10.1111/agec.12315

Durán Gil, C. A. (2017). Análisis espacial de las condiciones de vulnerabilidad social, económica, física y ambiental en el territorio colombiano. Perspectiva Geográfica, 22(1), 11-32. https:// doi.org/10.19053/01233769.5956

Engel, S. y Muller, A. (2016). Payments for environmental services to promote "climate-smart agriculture"? Potential and challenges. Agriculture Economics, 47(S1), 173-184. https://doi. org/10.1111/agec.12307

Febriani, L. y Lokantara I. G. W. (2017). Community participation towards the value of traditional architecture resilience, on the settlements' patters in Tenganan village, Amlapura. IOP Conference Series: Earth and EnvironmentalScience, 99(1), 1-9. https://doi.org/10.1088/1755$\underline{1315 / 99 / 1 / 012018}$

Gourdji, S., Mesa-Diez, J., Obando-Bonilla, D., Navarro-Racines, C., Moreno P., Fisher, M., Prager, S. y Ramirez-Villegas, J. (2016). Simulated near-term climate change impacts on major crops across Latin America and the Caribbean. American Geophysical Union, [abstract id. GC53F-06]. https://ui.adsabs.harvard.edu/abs/2016AGUFMGC53F..06G/abstract

Graffeo, M. (2017). Agriculture: Mitigation through food. Nature Climate Change, 7(4), 236-236. https://doi.org/10.1038/nclimate3269

Iglesias, A., Quiroga, S., Diz, A. y Garrote. L. (2011). Adapting agriculture to climate change. Economía Agraria y Recursos Naturales, 11(2), 109-122. https://doi.org/10.7201/ earn.2011.02.05 
Intergovernmental Panel on Climate Change. (2005). Guidance notes for lead aAuthors of the IPCC fourth assessment report on addressing uncertainties. https://wg1.ipcc.ch/publications/ supportingmaterial/uncertainty-guidance-note.pdf

Intergovernmental Panel on Climate Change. (2019). Special report global warming of $1,5{ }^{\circ} \mathrm{C}$. https://www.ipcc.ch/sr15/

Kammen, D. M. (2013). Complexity and interdisciplinary approaches to environmental research. Environmental Research Latters, 8(1), 1-4. https://doi.org/10.1088/1748-9326/8/1/010201

Kgosikoma, K. R., Lekota, P. y Kgosikoma, O. E. (2018). Agro-pastoralists' determinants of adaptation to climate change. International Journal of Climate Change Strategies and Management, 10(3), 488-500. https://doi.org/10.1108/IJCCSM-02-2017-0039

Leff, E. (2007). La complejidad ambiental, Polis 16, 1-11. http://journals.openedition.org/ polis/4605

Leff, E. (2004). Racionalidad ambiental. La reapropiación de la naturaleza. Siglo XXI.

Lopez, H., West, R., Dong, S., Goni, G., Kirtman, B., Lee, S-K. y Atlas, R. (2018). Early emergence of anthropogenically forced heat waves in the western United States and Great Lakes. Nature Climate Change, 8(5), 414-420. https://doi.org/10.1038/s41558-018-0116-y

Marsicek, J., Shuman, B. N., Bartlein, P. J., Shafer, S. L. y Brewer, S. (2018). Reconciling divergent trends and millennial variations in Holocene temperatures. Nature, 554(7690), 92-96. https://doi.org/10.1038/nature25464

Martín-Ortega, J. (2011). Costs of adaptation to climate change impacts on fresh-water systems: Existing estimates and research gaps. Agricultural and Resource Economics, 11(1), 5-28. https://doi.org/10.7201/earn.2011.01.01

Nangombe, S., Zhou, T., Zhang, W., Wu, B., Hu, S., Zou, L. y Li, D. (2018). Record-breaking climate extremes in Africa under stabilized $1.5^{\circ} \mathrm{C}$ and $2{ }^{\circ} \mathrm{C}$ global warming scenarios. Nature Climate Change, 8(5), 375-380. https://doi.org/10.1038/s41558-018-0145-6

Núñez, J. J., Carrero, D. M., Carvajal, J. C. y Mendoza, O. (2018). Assessing the impact of climate change on agriculture in Norte de Santander, Colombia. Journal of Physics: Conference Series, 1126(1), 012045. https://doi.org10.1088/1742-6596/1126/1/012045

Osorio García, S. N. (2012). El pensamiento complejo y la transdisciplinariedad: Fenómenos emergentes de una nueva racionalidad. Revista de la Facultad de Ciencias Económicas, 20(1), 262-291. https://doi.org/10.18359/rfce.2196 
http://doi.org/10.15359/ree.25-2.28

http://www.una.ac.cr/educare

educare@una.ac.cr

Pancorbo Jiménez, A. (2012). Cambio climático e incertidumbre: Un enfoque diferente en la información. En R. Mancinas Chávez (Coord.) y R. Fernández Reyes (Dir.), Jornadas internacionales, medios de comunicación y cambio climático (pp. 335-353). Facultad de Comunicación de la Universidad de Sevilla. https://idus.us.es/handle/114 41/36904

Peixoto, J. N. S., Nunes, M., Pereira Baliza, D., Parreiras Pereira, S. y Rosa, B. T. (2017). Cafeicultura familiar e as boas práticas agrícolas em bom sucesso - MG. Coffee Science, Lavras, 12(3), 365-373. https://doi.org/10.25186/cs.v12i3.1298

Rodríguez de Luque, J. J., González Rodríguez, C. E., Gourdji, S., Mason-D'Croz, D., ObandoBonilla, D., Mesa-Diez, J. y Prager, S. D. (2016). Impactos socioeconómicos del cambio climático en América Latina y el Caribe: 2020-2045. Cuadernos de Desarrollo Rural, 13(78), 11-34. https://doi.org/10.11144/Javeriana.cdr13-78.iscc

Saj, S., Torquebiau, E., Hainzelin, E., Pages, J.y Maraux, F. (2017).The way forward: An agroecological perspective for Climate-Smart Agriculture. Agriculture, Ecosystems and Environment, 250, 20-24. https://doi.org/10.1016/j.agee.2017.09.003

Soteriades, A. D., Murray-Rust, D., Trabucco, A. y Metzger, M. J. (2017). Understanding global climate change scenarios through bioclimate stratification. Environmental Research Letters, 12(8), 1-10. https://doi.org/10.1088/1748-9326/aa7689

Supadli, I., Saputri, A. y Mawengkang, H. (2018). Mitigating local natural disaster through social aware preparedness using complexity approach. IOP Conference Series: Earth and Environmental Science, 300(1), 1-7. https://doi.org/10.1088/1757-899X/300/1/012050

UN CC: Learn y Organización Mundial de la Salud. (2013). Módulo especializado en salud humana y cambio climático. https://unccelearn.org/course/view.php?id=30\&page=overview

Wilhelmi, O. V. y Hayden, M. H. (2010). Connecting people and place: A new framework for reducing urban vulnerability to extreme heat. Environmental. Research Letters, 5(1), 1-8. https://doi.org/10.1088/1748-9326/5/1/014021

Williams, B. K. y Johnson, F. A. (2013). Confronting dynamics and uncertainty in optimal decision making for conservation. Environmental. Research Letters, 8(2), 1-17. https://doi. org/10.1088/1748-9326/8/2/025004

Wyczykier, G. (2017). La problemática del desarrollo, las clases sociales y la burguesía en América Latina: Reflexiones conceptuales. Trabajo y sociedad, 29, 649-665. https://dialnet.unirioja. es/servlet/articulo?codigo $=6726910$ 